\title{
Research on the coordinated optimization operation method of Park Comprehensive Energy System Based on master-slave game
}

\author{
Nan $\mathrm{Xu}^{1}$; Bo Zhou ${ }^{1}$; Jing Nie ${ }^{1}$; Yan Song ${ }^{1}$; Zihao Zhao ${ }^{1}$ \\ ${ }^{1}$ State Grid Hebei Economic Research Institute, Hebei, 05000, China
}

\begin{abstract}
With the transformation of the energy market from the traditional vertical integrated structure to the interactive competitive structure, the distributed characteristics of the energy system become more and more obvious, and the traditional centralized optimization method is difficult to reveal the interaction between the multi-agent. In this paper, a method based on master-slave game is proposed to optimize the operation of park integrated energy system. Firstly, user load model, user benefit model, operator revenue and cost model are established for park integrated energy system. Secondly, the Stackelberg master-slave game model of interactive optimization operation is established, and the peak cutting compensation price is adjusted. Both of them aim at maximizing their own interests until the game equilibrium is achieved. A distributed cooperative optimization model with one master and many slaves is established and solved by the combination of genetic algorithm and quadratic programming. Finally, an example is given to verify the effectiveness of the proposed method.
\end{abstract}

\section{Introduction}

High efficiency, clean and low carbon are the main trend of energy development in the world. The park covers a variety of production capacity and energy users, with large load demand, complex energy consumption structure, low energy utilization efficiency and unreasonable energy structure. With the expansion of the scale of the park, the power supply pressure of the peak load in the park is further increased, and effective peak shaving means can enhance the power supply reliability in the park. In addition, when the peak load of the large power grid comes, if the park can cooperate with the external power grid to cut the peak load, it will be conducive to the safe and reliable operation of the power grid. A comprehensive energy system with combined cooling, heating and power (CCHP) system as the core unit will be built in the park, aiming to realize the reliable, efficient and clean energy consumption in the park, and realize the friendly interaction between the park and the large power grid. The interactive optimization of the park's comprehensive energy system can tap the demand response potential of all participants in the park, enhance the benign interaction between users and the power grid, and provide better comprehensive energy supply services for users.

In the process of energy transaction, the electricity selling company or energy operator shall first formulate price strategy according to the load demand, and then the user side shall make demand response according to the price information. The game process between the two has a sequence, which conforms to the dynamic game situation of the master-slave hierarchical structure. The master-slave game model should be used to analyze the interaction between the main bodies. In literature [1-3], the seller is regarded as the leader and the user as the follower. Based on the master-slave game model, the interaction mechanism between them in the power market is analyzed. In reference [4-5], aiming at a CCHP type community energy Internet, a pricing strategy for operators and a model for selecting the transaction mode of producers and consumers are established. Based on the master-slave game theory, a distributed energy management method is proposed. Literature [6] proposes an interactive optimization method of master-slave game for park comprehensive energy system by combining economic operation and auxiliary services. In reference [7], the distributed generation is regarded as the price receiver. Based on the master-slave game, the electricity price and power operation strategy of the operator are formulated, and the equipment output is guided by the price signal.

Therefore, this paper studies the operation strategy of Distributed Collaborative Optimization in the framework of master-slave game. The integrated energy retailer (IER) is the leader, and the new energy CCHP operators and load aggregators are the followers. At the same time, the pricing strategy, output plan and user demand of ier are optimized. This paper introduces the energy trading process and mathematical model, and proves that there is a unique Stackelberg equilibrium in the proposed game model, which is solved by combining genetic algorithm and two times programming. Finally, the correctness of the

\footnotetext{
*Corresponding author: 15931875763@163.com
} 
method is verified by an example.

\section{Comprehensive energy system structure of the park}

\subsection{System architecture}

In this paper, IER is proposed based on the concept of electricity selling company in the electricity market. On the basis of electricity trading, thermal energy trading is considered to meet the diversified needs of users. As a bridge between the source and the load, IER optimizes the purchase and sale price of electricity and heat based on the relationship between supply and demand, purchases energy such as electricity and heat from the energy supply side, and sells it to the energy consumption side to earn income.

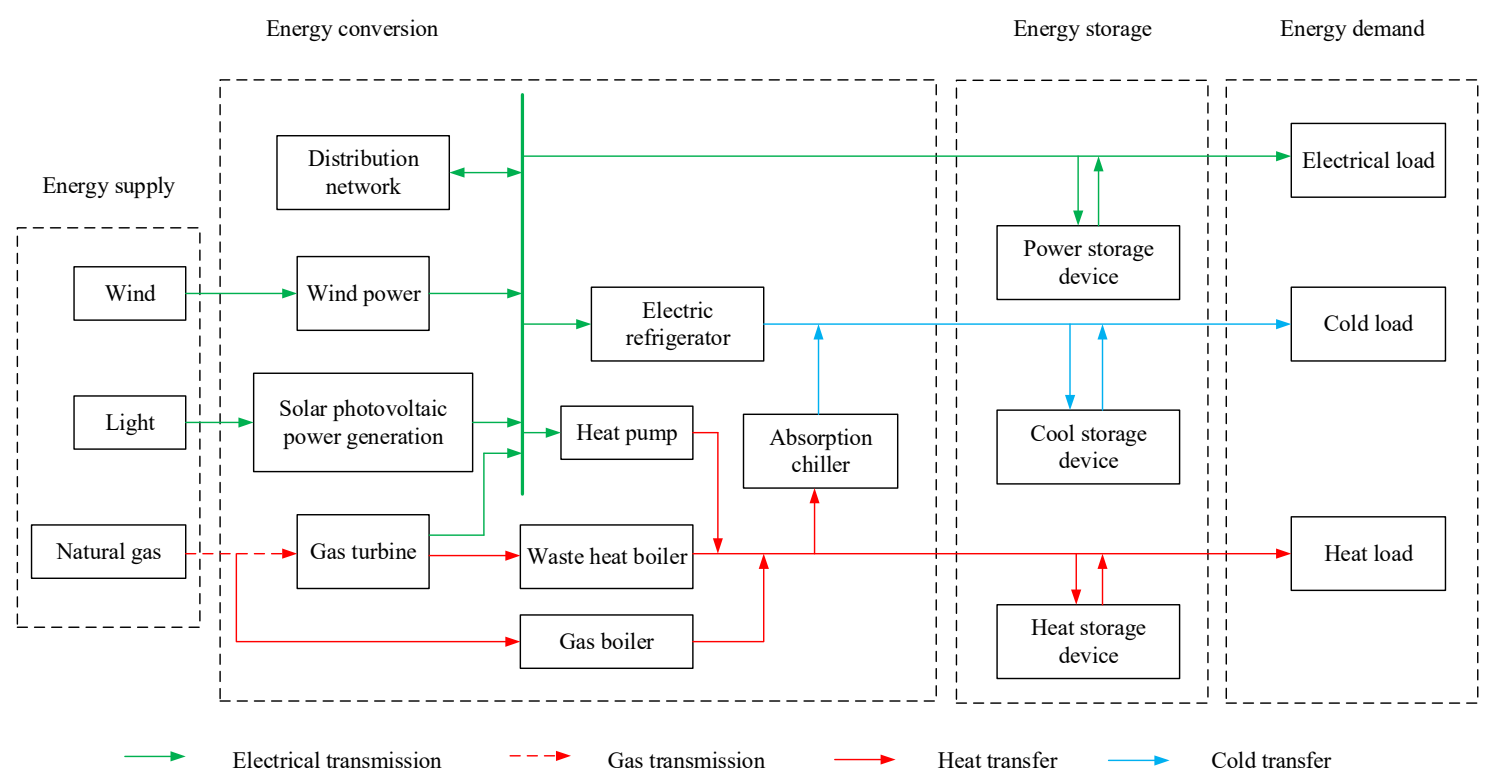

Figure 1. Comprehensive energy map of the park

\subsection{Energy trading process}

Energy trading includes two stages: pricing decision and quantitative decision. The two stages have order, influence each other, and cycle until equilibrium. Stage 1 (pricing): the upper tier IER formulates the purchase and sale price and hot price according to the supply-demand relationship and market information to maximize its own income. Stage 2 (Quantitative): the power supply side and the power consumption side of the lower layer determine the optimal output and load demand respectively according to the price signal of IER, so the optimal decision of the lower layer can be regarded as a function of the decision variables of the upper layer.

\section{Master-slave game framework}

\subsection{Basic concepts}

According to the above description, the optimization of the energy supply side and the energy consumption side is based on the IER quotation, and their optimization results will react on the IER quotation. This energy transaction process is in line with the dynamic game situation of the master-slave hierarchical structure. Therefore, this paper takes IER as the leader, operators and load aggregators as different followers, and establishes a Stackelberg blog with one master and many slaves The chess model can be expressed as follows:

$$
G=\left\{N ; \rho_{\text {ier }} ;\left\{\delta_{c}, \delta_{\text {user }}\right\} ; F_{i e r} ;\left\{F_{c}, F_{\text {user }}\right\}\right\}
$$

The above game model includes three elements: participants, strategies and benefits, which are specifically expressed as follows:

1) Participants: IER, operator and load aggregator are the three participants of the game, and the set of participants is represented as $N=\{$ ier, c, user $\}$.

2) Strategy: the strategy of leader IER is the 24-hour purchase and sale price and heat price, which can be expressed as $\rho_{i e r}=\left(c_{e, s}, c_{h, s}, c_{e, b}, c_{\mathrm{h}, b}\right)$ in the form of vector; the strategy of new energy CCHP is the output power of internal combustion generator and boiler at each time, which can be expressed as vector $\delta_{c c h p}=\left(P_{I}, Q_{B}\right) ;$ the strategy of load aggregator is the power that can level off the power load and reduce the heat load at each time, which can be expressed as vector $\delta_{\text {user }}=\left(P_{e}, Q_{c}\right)$

\subsection{Stackelberg equilibrium}

When all followers make the optimal response according to the leader's strategy, and the leader also accepts this response, the game reaches Stackelberg equilibrium. If $\left(\rho_{\text {ier }}{ }^{*}, \delta_{c}^{*}, \delta_{\text {user }}{ }^{*}\right)$

is the equilibrium solution of the 
CIES master-slave game in this article, the following conditions must be met:

$$
\begin{aligned}
& F_{\text {ier }}\left(\rho_{\text {ier }}{ }^{*}, \delta_{c}^{*}, \delta_{\text {user }}{ }^{*}\right) \geq F_{\text {ier }}\left(\rho_{\text {ier }}, \delta_{c}^{*}, \delta_{\text {user }}{ }^{*}\right) \\
& F_{c}\left(\rho_{\text {ier }}{ }^{*}, \delta_{c}^{*}, \delta_{\text {user }}{ }^{*}\right) \geq F_{c}\left(\rho_{\text {ier }}{ }^{*}, \delta_{c}, \delta_{\text {user }}^{*}\right) \\
& F_{\text {user }}\left(\rho_{\text {ier }}^{*}, \delta_{c}^{*}, \delta_{\text {user }}^{*}\right) \geq F_{\text {user }}\left(\rho_{\text {ier }}{ }^{*}, \delta_{c}^{*}, \delta_{\text {user }}\right)
\end{aligned}
$$

In Stackelberg's equilibrium solution, neither party can gain greater benefits by unilaterally changing strategies. Before solving the Stackelberg equilibrium solution, we need to prove its existence and uniqueness, using the following theorem.

Theorem: When the master-slave game model meets the following conditions, there is a unique Stackelberg equilibrium:

1) The leader and the following strategy set are nonempty tight convex sets;

2) When the leader's strategy is given, all followers have a unique optimal solution;

3) When the strategy of the follower is given, the leader has a unique optimal solution.

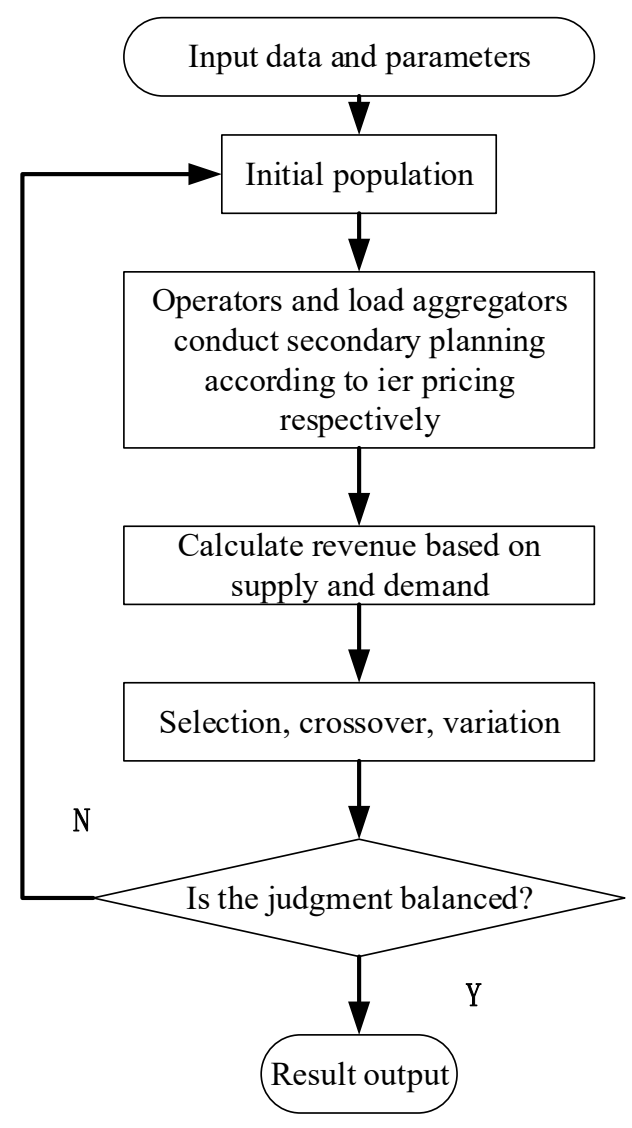

Figure 2. GA-PQ algorithm flow chart

\section{Case study}

Taking a certain park as an example, the collaborative optimization operation method proposed in this paper is simulated and analyzed. Because the energy trading mode of winter conditions is similar to that of summer conditions, this paper only simulates and analyzes the operation and trading process of electricity and heat flow under winter conditions. The peak of electric load appears at 12:00 and 18:00-22:00, and the peak of thermal load appears at 4:00-8:00. Assuming that the translatable electric load and the thermal load can be reduced by $20 \%$ of the total load, the upper limit of the translatable electric load power is $240 \mathrm{~kW}$.

The convergence trends of leaders and followers are different. With the increase of the number of iterations, the income of IER gradually increases, and the income of new energy CCHP operators and the residual value of consumers gradually decrease. When the Stackelberg equilibrium is reached, their strategy no longer changes, indicating that under this strategy, no participant can gain more benefits by independently changing the strategy. In the end, the revenue of operators and IER were 8254 and 5783 yuan, respectively, and the consumer surplus stabilized at 11137 yuan.

The waste heat of the gas generator is supplied with the gas boiler, and the waste heat is directly related to the power generation of the gas generator. In order to ensure the supply of heat energy and avoid paying penalties, IER guides the boiler's output by adjusting the purchase price of heat to achieve a balance between supply and demand. When the new energy CCHP operator operates in the traditional electric heating mode, the revenue is 7765 yuan. If the optimized operation method proposed in this article is used, the revenue will increase to 8254 yuan.

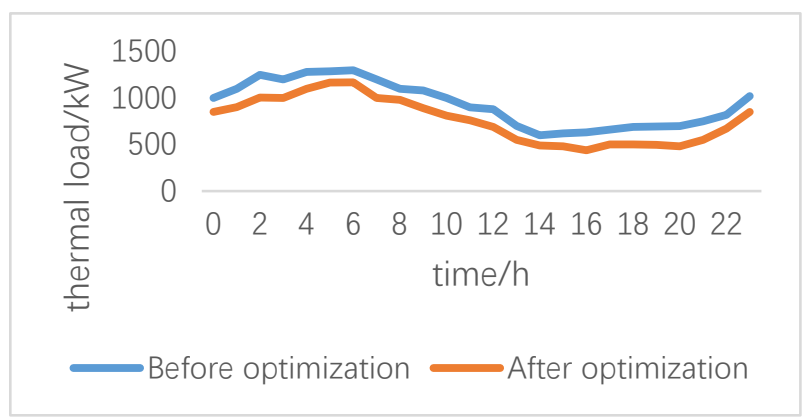

Figure 3. Load aggregator optimization results

\section{Conclusion}

1) Through theoretical analysis and derivation, it is proved that the proposed CIES master-slave game model has a unique Stackelberg equilibrium solution.

2) The simulation results of the example illustrate the method in this paper. The price signal can guide the output and adjust the user's energy consumption plan, which can provide energy-side benefits. At the same time, the energy consumption cost can be reduced and the load fluctuation can be suppressed under the premise of ensuring energy consumption satisfaction.

The energy storage device has not been considered in this paper. The direct interaction between the IER and the power grid will result in a decrease in its revenue. In the future research, the impact of the energy storage device on the CIES energy transaction needs to be further considered to realize the "source-charge-storage" collaborative optimized operation. 


\section{Acknowledgments}

This research was funded by the Science and Technology Project of the State Grid Corporation of China. The name of the project is the research of market integration transaction and typical business production simulation and benefit evaluation method of the integrated energy service system in Xiongan New District (Project No. SGHEJY00JJJS1900017).

\section{References}

1. Wei Wei, Liu Feng, Mei Shengwei. Energy pricing and dispatch for smart grid retailers under demand response and market price uncertainty[J]. IEEE Transactions on Smart Grid,2015,6(3):1364-1374.

2. PaudelA, Chaudhari K, Long Chao, et al. Peer-to-peer energy trading in a prosumer-based community microgrid: a game-theoretic model[J]. IEEE Transactions on Industrial electronics,2019,66(60876097).

3. Maharjan S, Zhu Quanyan, Zhang Yan, et al. Dependable demand response management in the smart grid: a Stackelberg game approach[J]. IEEE Transactions on Smart Grid,2013,4(1):120-132.

4. Ma Li, Liu Nian, Zhang Jianhua, et al. Distributed energy management of community energy internet based on leader-followers game[J]. Power System Technology,2016(12):41-48(in Chinese).

5. Liu Nian, $\mathrm{He} \mathrm{Li}, \mathrm{Yu}$ Xinghong, et al. Multiparty energy management for grid-connected microgrids with heat-and electricity-coupled demand response[J]. IEEE Transactions on Industrial Informatics, 2018,14(5):1887-1897.

6. Zhou Changchen, Ma Xiyuan, Guo Xiaobin, et al. Leader-follower game based optimized operation method for interaction of integrated energy system in industrial park $[\mathrm{J}]$. Automation of Electric Power Systems,2019,43(07):111-121(in Chinese).

7. Zhao Wenhui, Qi Yu, Fan Hanlu. Stackelberg game scheduling for regional power grids [J]. Control Theory and Technology,2018,35(05):71-79(in Chinese).

8. Ju Liwei, Tan Zhongfu, Li Huanhuan, et al. Multiobjective operation optimization and evaluation model for CCHP and renewable energy based hybrid energy system driven by distributed energy resources in China[J]. Energy,2016,111:322-340. 